\title{
THE EXTENDED $Z_{N^{-}}$TODA HIERARCHY
}

\author{
CHUANZHONG LI $\dagger$, JINGSONG HE $\ddagger$ \\ Department of Mathematics and Ningbo Collabrative Innovation Center of Nonlinear Harzard System of \\ Ocean and Atmosphere, \\ Ningbo university, Ningbo 315211, China, \\ †lichuanzhong@nbu.edu.cn \\ $\ddagger$ †hejingsong@nbu.edu.cn
}

\begin{abstract}
The extended flow equations of a new $Z_{N}$-Toda hierarchy which takes values in a commutative subalgebra $Z_{N}$ of $g l(N, \mathbb{C})$ is constructed. Meanwhile we give the Hirota bilinear equations and tau function of this new extended $Z_{N}$-Toda hierarchy(EZTH). Because of logarithm terms, some extended Vertex operators are constructed in generalized Hirota bilinear equations which might be useful in topological field theory and Gromov-Witten theory. Meanwhile the Darboux transformation and bi-hamiltonian structure of this hierarchy are given. From hamiltonian tau symmetry, we give another different tau function of this hierarchy with some unknown mysterious connections with the one defined from the point of Sato theory.
\end{abstract}

Mathematics Subject Classifications(2000). 37K05, 37K10, 37K20.

Keywords: extended $Z_{N}$-Toda hierarchy, Hirota quadratic equation, Darboux transformation, Bi-hamiltonian structure.

\section{Contents}

1. Introduction

2. Factorization and Logarithm operators

3. Lax equations of EZTH

3.1. The extended $Z_{N^{-}}$Toda equations

4. Hirota bilinear equations

5. Tau-functions of EZTH

6. Generalized matrix Vertex operators and Hirota quadratic equations

7. Darboux transformations of the EZTH

7.1. Soliton solutions

8. Bi-Hamiltonian structure and tau symmetry

9. Conclusions and Discussions

References

\section{INTRODUCTION}

The KP hierarchy and Toda lattice hierarchy as completely integrable systems have many important applications in mathematics and physics including the theory of Lie algebra representation, orthogonal polynomials and random matrix model [1 5]. KP and Toda systems have many kinds of reduction or extension, for example BKP, CKP hierarchy, extended Toda 
hierarchy (ETH) [6, 7], bigraded Toda hierarchy (BTH) [8]- [14] and so on. There are another kinds of generalization called multi-component KP [15, 16] or multi-component Toda systems which attract more and more attention because its widely use in many fields such as the fields of multiple orthogonal polynomials and non-intersecting Brownian motions.

The multicomponent KP hierarchy was discussed with application on representation theory and random matrix model in [15, 16]. In [3], it was noticed that $\tau$ functions of a $2 N$ multicomponent KP provide solutions of the $N$-multicomponent 2D Toda hierarchy. The multicomponent 2D Toda hierarchy was considered from the point of view of the Gauss-Borel factorization problem, the theory of multiple matrix orthogonal polynomials, non-intersecting Brownian motions and matrix Riemann-Hilber problem [17]- 20]. In fact the multicomponent 2D Toda hierarchy in [18] is a periodic reduction of bi-infinite matrix-formed two dimensional Toda hierarchy. The coefficients(or dynamic variables) of the multicomponent 2D Toda hierarchy take values in complex finite-sized matrix. The multicomponent 2D Toda hierarchy contains matrix-formed Toda equation as the first flow equation.

Adding additional logarithm flows to the Toda lattice hierarchy, it becomes the extended Toda hierarchy [6] defined on a Lax operator

$$
L=\Lambda+u+e^{v} \Lambda^{-1}, \quad u, v \in \mathbb{C},
$$

which governs the Gromov-Witten invariant of $C P^{1}$. The Gromov-Witten potential of $C P^{1}$ is actually a tau function of the extended Toda hierarchy, i.e. the Gromov-Witten potential $\tau$ of $C P^{1}$ makes the following Hirota quadratic equations [7] of the ETH

$$
\frac{d \lambda}{\lambda}\left(\Gamma^{\delta \#} \otimes \Gamma^{\delta}\right)\left(\Gamma^{\alpha} \otimes \Gamma^{-\alpha}-\Gamma^{-\alpha} \otimes \Gamma^{\alpha}\right)(\tau \otimes \tau)
$$

regular in $\lambda$ computed at $q_{0}-q_{0}^{\prime}=l \epsilon$ for each $l \in \mathbb{Z}$. The extended bigraded Toda hierarchy (EBTH) is the extension of the bigraded Toda hierarchy (BTH) which includes additional logarithm flows [8, 10]. This paper will tell us that the Hirota quadratic equations(1.2) can be derived as a reduction on Lie algebra from the Hirota bilinear equation of the extended $Z_{N}$-Toda hierarchy. Therefore the application of our Hirota bilinear equation of the extended $Z_{N}$-Toda hierarchy in Gromov-Witten theory becomes a great motivation of our study. The Hirota bilinear equation of EBTH was equivalently constructed in our early paper [9] and a very recent paper [21], because of the equivalence of $t_{1, N}$ flow and $t_{0, N}$ flow of EBTH. Meanwhile it was proved to govern Gromov-Witten invariant of the total descendent potential of $\mathbb{P}^{1}$ orbifolds [21]. A nature question is what about the corresponding extended multi-component Toda hierarchy ( as a matrix-formed generalization of extended Toda hierarchy [6]) and extended multicomponent bigraded Toda hierarchy. There is a class of orbifolds which should be governed by some logarithmic hierarchies. That is why we think this kind of new logarithmic hierarchy might be useful in Gromov-Witten invariants theory governed by these two new hierarchies. With this motivation, our paper [22] will be devoted to construct a kind of Hirota quadratic equation taking values in a differential matrix algebra set. This kind of Hirota bilinear equation might be useful in Gromov-Witten theory. In [23], a new hierarchy called as $Z_{m}$-KP hierarchy which take values in a maximal commutative subalgebra of $g l(m, \mathbb{C})$ was constructed, meanwhile the relation between Frobenius manifold and dispersionless reduced $Z_{m}$-KP hierarchy was discussed. This inspires us to consider the Hirota quadratic equation of the commutative version of extended multi-component Toda hierarchy which might be useful in Frobenius manifold theory in this paper. 
This paper is arranged as follows. In the next section we recall factorization problem and construct the logarithm matrix operators using which we define the extended flow of the multicomponent $Z_{N^{-}}$Toda hierarchy. In Section 3, we will give the Lax equations of the extended $Z_{N}$-Toda hierarchy $(\mathrm{EZTH})$, meanwhile the multicomponent $Z_{N^{-}}$Toda equations and the extended equations are introduced in this hierarchy. By Sato equations, Hirota bilinear equations of the EZTH are proved in Section 4. The tau function of the EZTH will be defined in Section 5 which lead to the formalism of the generalized matrix Vertex operators and Hirota quadratic equations in Section 6. In section 7, multi-fold transformations of the EZTH will be constructed using determinant technique in [25]. To prove the integrability of this new hierarchy, Bi-hamiltonian structure and tau symmetry of the EZTH are constructed in Section 8. Section 9 will be devoted to a short conclusions and discussions.

\section{FACTORIZATION AND LOGARITHM OPERATORS}

Suppose that $\tilde{G}$ is a group which contains linear invertible elements of complex $N \times N$ complex matrices and its Lie algebra $\tilde{\mathfrak{g}}$ denotes the associative algebra of complex $N \times N$ complex matrices $M_{N}(\mathbb{C})$. Now we will consider the linear space of function $g: \mathbb{R} \rightarrow M_{N}(\mathbb{C})$ with the shift operator $\Lambda$ acting on these functions as $(\Lambda g)(x):=g(x+\epsilon)$. A Left multiplication by $X: \mathbb{R} \rightarrow M_{N}(\mathbb{C})$ is as $X \Lambda^{j},\left(X \Lambda^{j}\right)(g)(x):=X(x) \circ g(x+j \epsilon)$ with defining the product $\left(X(x) \Lambda^{i}\right) \circ\left(Y(x) \Lambda^{j}\right):=X(x) Y(x+i \epsilon) \Lambda^{i+j}$. Then the set $\mathfrak{g}$ of Laurent series in $\Lambda$ as an associative algebra is a Lie algebra under the standard commutator.

This Lie algebra has the following important splitting

$$
\mathfrak{g}=\mathfrak{g}_{+} \oplus \mathfrak{g}_{-},
$$

where

$$
\mathfrak{g}_{+}=\left\{\sum_{j \geq 0} X_{j}(x) \Lambda^{j}, \quad X_{j}(x) \in M_{N}(\mathbb{C})\right\}, \quad \mathfrak{g}_{-}=\left\{\sum_{j<0} X_{j}(x) \Lambda^{j}, \quad X_{j}(x) \in M_{N}(\mathbb{C})\right\} .
$$

The splitting (2.1) leads us to consider the following factorization of $g \in G$

$$
g=g_{-}^{-1} \circ g_{+}, \quad g_{ \pm} \in G_{ \pm}
$$

where $G_{ \pm}$have $\mathfrak{g}_{ \pm}$as their Lie algebras. $G_{+}$is the set of invertible linear operators of the form $\sum_{j \geq 0} g_{j}(x) \Lambda^{j}$; while $G_{-}$is the set of invertible linear operators of the form $1+\sum_{j<0} g_{j}(x) \Lambda^{j}$. This algebra has a maximal commutative subalgebra $Z_{N}=\mathbb{C}[\Gamma] /\left(\Gamma^{N}\right)$ and $\Gamma=\left(\delta_{i, j+1}\right)_{i j} \in$ $g l(N, \mathbb{C})$. Denote $Z_{N}(\Lambda):=\mathfrak{g}_{c}$, then we have the following splitting

$$
\mathfrak{g}_{c}=\mathfrak{g}_{c+} \oplus \mathfrak{g}_{c-},
$$

where

$$
\mathfrak{g}_{c+}=\left\{\sum_{j \geq 0} X_{j}(x) \Lambda^{j}, \quad X_{j}(x) \in Z_{N}\right\}, \quad \mathfrak{g}_{c-}=\left\{\sum_{j<0} X_{j}(x) \Lambda^{j}, \quad X_{j}(x) \in Z_{N}\right\} .
$$

The splitting (2.3) leads us to consider the following factorization of $g_{c} \in G_{c}$

$$
g_{c}=g_{c-}^{-1} \circ g_{c+}, \quad g_{c \pm} \in G_{c \pm},
$$

where $G_{c \pm}$ have $\mathfrak{g}_{c \pm}$ as their Lie algebras. $G_{c+}$ is the set of invertible linear operators of the form $\sum_{j \geq 0} g_{j}(x) \Lambda^{j}$; while $G_{c-}$ is the set of invertible linear operators of the form $1+\sum_{j<0} g_{j}(x) \Lambda^{j}$. 
Now we introduce the following free operators $W_{0}, \bar{W}_{0} \in G_{c}$

$$
\begin{aligned}
& W_{0}:=\mathrm{e}^{\sum_{j=0}^{\infty} t_{j} \frac{\Lambda^{j}}{\epsilon j !}+s_{j} \frac{\Lambda^{j}}{\epsilon j !}\left(\epsilon \partial-c_{j}\right)}, \quad \partial=\frac{\partial}{\partial x} \\
& \bar{W}_{0}:=\mathrm{e}^{\sum_{j=0}^{\infty} t_{j} \frac{\Lambda^{-j}}{\epsilon j !}+s_{j} \frac{\Lambda^{-j}}{\epsilon j !}\left(\epsilon \partial-c_{j}\right)}, \quad c_{j}=\sum_{i=1}^{j} \frac{1}{i},
\end{aligned}
$$

where $t_{j}, s_{j} \in \mathbb{C}$ will play the role of continuous times.

We define the dressing operators $W, \bar{W}$ as follows

$$
W:=S \circ W_{0}, \quad \bar{W}:=\bar{S} \circ \bar{W}_{0}, \quad S \in G_{c-}, \bar{S} \in G_{c+} .
$$

Given an element $g \in G_{c}$ and denote $t=\left(t_{j}\right), s=\left(s_{j}\right) ; j \in \mathbb{N}$, one can consider the factorization problem in $G_{c}$ similarly as [18]

$$
W \circ g=\bar{W},
$$

i.e. the factorization problem

$$
S(t, s) \circ W_{0} \circ g=\bar{S}(t, s) \circ \bar{W}_{0} .
$$

Observe that $S, \bar{S}$ have expansions of the form

$$
\begin{aligned}
& S=\mathbb{I}_{N}+\omega_{1}(x) \Lambda^{-1}+\omega_{2}(x) \Lambda^{-2}+\cdots \in G_{c-}, \\
& \bar{S}=\bar{\omega}_{0}(x)+\bar{\omega}_{1}(x) \Lambda+\bar{\omega}_{2}(x) \Lambda^{2}+\cdots \in G_{c+} .
\end{aligned}
$$

Also we define the symbols of $S, \bar{S}$ as $\mathbb{S}, \overline{\mathbb{S}}$

$$
\begin{aligned}
& \mathbb{S}=\mathbb{I}_{N}+\omega_{1}(x) \lambda^{-1}+\omega_{2}(x) \lambda^{-2}+\cdots, \\
& \overline{\mathbb{S}}=\bar{\omega}_{0}(x)+\bar{\omega}_{1}(x) \lambda+\bar{\omega}_{2}(x) \lambda^{2}+\cdots .
\end{aligned}
$$

The inverse operators $S^{-1}, \bar{S}^{-1}$ of operators $S, \bar{S}$ have expansions of the form

$$
\begin{aligned}
& S^{-1}=\mathbb{I}_{N}+\omega_{1}^{\prime}(x) \Lambda^{-1}+\omega_{2}^{\prime}(x) \Lambda^{-2}+\cdots \in G_{c-}, \\
& \bar{S}^{-1}=\bar{\omega}_{0}^{\prime}(x)+\bar{\omega}_{1}^{\prime}(x) \Lambda+\bar{\omega}_{2}^{\prime}(x) \Lambda^{2}+\cdots \in G_{c+} .
\end{aligned}
$$

Also we define the symbols of $S^{-1}, \bar{S}^{-1}$ as $\mathbb{S}^{-1}, \overline{\mathbb{S}}^{-1}$

$$
\begin{aligned}
& \mathbb{S}^{-1}=\mathbb{I}_{N}+\omega_{1}^{\prime}(x) \lambda^{-1}+\omega_{2}^{\prime}(x) \lambda^{-2}+\cdots, \\
& \overline{\mathbb{S}}^{-1}=\bar{\omega}_{0}^{\prime}(x)+\bar{\omega}_{1}^{\prime}(x) \lambda+\bar{\omega}_{2}^{\prime}(x) \lambda^{2}+\cdots .
\end{aligned}
$$

The Lax operators $\mathcal{L} \in G_{c}$ are defined by

$$
\mathcal{L}:=W \circ \Lambda \circ W^{-1}=\bar{W} \circ \Lambda^{-1} \circ \bar{W}^{-1},
$$

and have the following expansions

$$
\mathcal{L}=\Lambda+u_{1}(x)+u_{2}(x) \Lambda^{-1}
$$

In fact the Lax operators $\mathcal{L} \in G_{c}$ can also be equivalently defined by

$$
\mathcal{L}:=S \circ \Lambda \circ S^{-1}=\bar{S} \circ \Lambda^{-1} \circ \bar{S}^{-1} .
$$

These definitions are continuous interpolated version of the multi-component commutative Toda hierarchy, i.e. a continuous spatial parameter $x$ was brought into this hierarchy. Under this 
meaning, the continuous flow $\frac{\partial}{\partial x}$ is missing. To make these flows complete, we define the following logarithm matrix

(2.18) $\log _{-} \mathcal{L}=-\bar{W} \circ \epsilon \partial \circ \bar{W}^{-1}=-\bar{S} \circ \epsilon \partial \circ \bar{S}^{-1}$,

where $\partial$ is the derivative about spatial variable $x$.

Combining these above logarithm operators together can derive following important logarithm matrix

$$
\log \mathcal{L}:=\frac{1}{2}\left(\log _{+} \mathcal{L}+\log _{-} \mathcal{L}\right)=\frac{1}{2}\left(S \circ \epsilon \partial \circ S^{-1}-\bar{S} \circ \epsilon \partial \circ \bar{S}^{-1}\right):=\sum_{i=-\infty}^{+\infty} W_{i} \Lambda^{i} \in G_{c}
$$

which will generate a series of flow equations which contain the spatial flow in later defined Lax equations.

\section{LAX EQUATIONS OF EZTH}

In this section we will use the factorization problem (2.8) to derive Lax equations. Let us first introduce some convenient notations.

Definition 1. Matrix operators $B_{j}, D_{j}$ are defined as follows

$$
B_{j}:=\frac{\mathcal{L}^{j+1}}{(j+1) !}, \quad D_{j}:=\frac{2 \mathcal{L}^{j}}{j !}\left(\log \mathcal{L}-c_{j}\right), \quad c_{j}=\sum_{i=1}^{j} \frac{1}{i}, j \geq 0 .
$$

Now we give the definition of the extended $Z_{N}$-Toda hierarchy(EZTH).

Definition 2. The extended $Z_{N}$-Toda hierarchy is a hierarchy in which the dressing operators $S, \bar{S}$ satisfy the following Sato equations

$$
\begin{array}{llrl}
\epsilon \partial_{t_{j}} S=-\left(B_{j}\right)_{-} S, & \epsilon \partial_{t_{j}} \bar{S}=\left(B_{j}\right)_{+} \bar{S}, \\
\epsilon \partial_{s_{j}} S=-\left(D_{j}\right)_{-} S, & \epsilon \partial_{s_{j}} \bar{S}=\left(D_{j}\right)_{+} \bar{S} .
\end{array}
$$

Then one can easily get the following proposition about $W, \bar{W}$.

Proposition 1. The dressing operators $W, \bar{W}$ are subject to the following Sato equations

$$
\begin{array}{rlrl}
\epsilon \partial_{t_{j}} W & =\left(B_{j}\right)_{+} W, & \epsilon \partial_{t_{j}} \bar{W}=\left(B_{j}\right)_{+} \bar{W}, \\
\epsilon \partial_{s_{j}} W=\left(\frac{\mathcal{L}^{j}}{j !}\left(\log _{+} \mathcal{L}-c_{j}\right)-\left(D_{j}\right)_{-}\right) W, & \epsilon \partial_{s_{j}} \bar{W}=\left(-\frac{\mathcal{L}^{j}}{j !}\left(\log _{-} \mathcal{L}-c_{j}\right)+\left(D_{j}\right)_{+}\right) \bar{W} .
\end{array}
$$

From the previous proposition we derive the following Lax equations for the Lax operators.

Proposition 2. The Lax equations of the EZTH are as follows

$$
\begin{aligned}
& \epsilon \partial_{t_{j}} \mathcal{L}=\left[\left(B_{j}\right)_{+}, \mathcal{L}\right], \quad \epsilon \partial_{s_{j}} \mathcal{L}=\left[\left(D_{j}\right)_{+}, \mathcal{L}\right], \epsilon \partial_{t_{j}} \log \mathcal{L}=\left[\left(B_{j}\right)_{+}, \log \mathcal{L}\right], \\
& \epsilon(\log \mathcal{L})_{s_{j}}=\left[-\left(D_{j}\right)_{-}, \log _{+} \mathcal{L}\right]+\left[\left(D_{j}\right)_{+}, \log _{-} \mathcal{L}\right] .
\end{aligned}
$$

To see this kind of hierarchy more clearly, the $Z_{N}$-Toda equations as the $t_{0}$ flow equations will be given in next subsection. 
3.1. The extended $Z_{N}$-Toda equations. As a consequence of the factorization problem (2.8) and Sato equations, after taking into account that $S \in G_{c-}$ and $\bar{S} \in G_{c+}$, the $t_{0}$ flow of $\mathcal{L}$ in the form of $\mathcal{L}=\Lambda+U+V \Lambda^{-1}$ is as

$$
\epsilon \partial_{t_{0}} \mathcal{L}=\left[\Lambda+U, V \Lambda^{-1}\right]
$$

which lead to matrix-Toda equation

$$
\begin{aligned}
\epsilon \partial_{t_{0}} U & =V(x+\epsilon)-V(x), \\
\epsilon \partial_{t_{0}} V & =U(x) V(x)-V(x) U(x-\epsilon) .
\end{aligned}
$$

Of course, one can switch the order of the matrices because of the commutativity of $Z_{N}$. Suppose

$$
U=\left[\begin{array}{cc}
u_{0} & 0 \\
u_{1} & u_{0}
\end{array}\right], \quad V=\left[\begin{array}{cc}
v_{0} & 0 \\
v_{1} & v_{0}
\end{array}\right],
$$

then the specific coupled Toda equation is

$$
\begin{aligned}
\epsilon \partial_{t_{0}} u_{0} & =v_{0}(x+\epsilon)-v_{0}(x) \\
\epsilon \partial_{t_{0}} u_{1} & =v_{1}(x+\epsilon)-v_{1}(x) \\
\epsilon \partial_{t_{0}} v_{0} & =u_{0}(x) v_{0}(x)-v_{0}(x) v_{0}(x-\epsilon) \\
\epsilon \partial_{t_{0}} v_{1} & =\left(u_{1}(x)-u_{1}(x-\epsilon)\right) v_{0}(x)-v_{1}(x)\left(u_{0}(x)-u_{0}(x-\epsilon) .\right.
\end{aligned}
$$

To get the standard matix-Toda equation, one need to use the alternative expressions

$$
\begin{aligned}
& U:=\omega_{1}(x)-\omega_{1}(x+\epsilon)=\epsilon \partial_{t_{1}} \phi(x), \\
& V=\mathrm{e}^{\phi(x)} \mathrm{e}^{-\phi(x-\epsilon)}=-\epsilon \partial_{t_{1}} \omega_{1}(x) .
\end{aligned}
$$

From Sato equation we deduce the following set of nonlinear partial differential-difference equations

$$
\left\{\begin{aligned}
\omega_{1}(x)-\omega_{1}(x+\epsilon) & =\epsilon \partial_{t_{1}}\left(\mathrm{e}^{\phi(x)}\right) \cdot \mathrm{e}^{-\phi(x)} \\
\epsilon \partial_{t_{1}} \omega_{1}(x) & =-\mathrm{e}^{\phi(x)} \mathrm{e}^{-\phi(x-\epsilon)}
\end{aligned}\right.
$$

Observe that if we cross the two first equations, then we get

$$
\epsilon^{2} \partial_{t_{1}}^{2} \phi(x)=\mathrm{e}^{\phi(x+\epsilon)} \mathrm{e}^{-\phi(x)}-\mathrm{e}^{\phi(x)} \mathrm{e}^{-\phi(x-\epsilon)}
$$

which is the $N \times N$ matrix-valued extension of the Toda equation, from which the original Toda equation appears for $N=1$. When $N=2$, the equation for $\phi=\left[\begin{array}{cc}\phi_{0} & 0 \\ \phi_{1} & \phi_{0}\end{array}\right]$ is the following coupled Toda system

$$
\begin{aligned}
& \epsilon^{2} \partial_{t_{1}}^{2} \phi_{0}(x)=\mathrm{e}^{\phi_{0}(x+\epsilon)-\phi_{0}(x)}-\mathrm{e}^{\phi_{0}(x)-\phi_{0}(x-\epsilon)}, \\
& \epsilon^{2} \partial_{t_{1}}^{2} \phi_{1}(x)=\left(\phi_{1}(x+\epsilon)-\phi_{1}(x)\right) \mathrm{e}^{\phi_{0}(x+\epsilon)-\phi_{0}(x)}-\left(\phi_{1}(x)-\phi_{1}(x-\epsilon)\right) \mathrm{e}^{\phi_{0}(x)-\phi_{0}(x-\epsilon)} .
\end{aligned}
$$

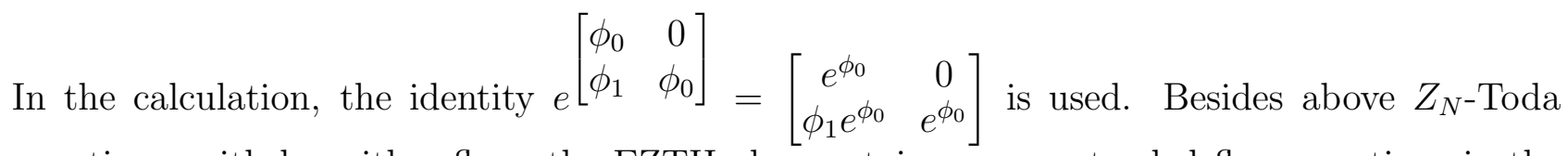
equations, with logarithm flows the EZTH also contains some extended flow equations in the 
next part. Here we consider the extended flow equations in the simplest case, i.e. the $s_{0}$ flow for $\mathcal{L}=\Lambda+u_{0}+u_{1} \Lambda^{-1}$,

$$
\begin{aligned}
\epsilon \partial_{s_{0}} \mathcal{L} & =\left[\left(S \epsilon \partial_{x} S^{-1}\right)_{+}, \mathcal{L}\right] \\
& =\left[\epsilon \partial_{x} S S^{-1}, \mathcal{L}\right] \\
& =\epsilon \mathcal{L}_{x}
\end{aligned}
$$

which leads to the following specific equation

$$
\partial_{s_{0}} U=U_{x}, \quad \partial_{s_{0}} V=V_{x}
$$

To see the extended equations clearly, one need to rewrite the extended flows in the Lax equations of the EZTH as in the following lemma.

Lemma 1. The extended flows in Lax formulation of the EZTH can be equivalently given by

$$
\begin{aligned}
& \epsilon \frac{\partial \mathcal{L}}{\partial s_{j}}=\left[D_{j}, \mathcal{L}\right] \\
& D_{j}=\left(\frac{\mathcal{L}^{j}}{j !}\left(\log _{+} \mathcal{L}-c_{j}\right)\right)_{+}-\left(\frac{\mathcal{L}^{j}}{j !}\left(\log _{-} \mathcal{L}-c_{j}\right)\right)_{-}
\end{aligned}
$$

which can also be rewritten in the form

$$
\begin{aligned}
& \epsilon \frac{\partial \mathcal{L}}{\partial s_{n}}=\left[\bar{D}_{n}, \mathcal{L}\right] \\
& \bar{D}_{j}=\frac{\mathcal{L}^{j}}{j !} \epsilon \partial+\left[\frac{\mathcal{L}^{j}}{j !}\left(\sum_{k<0} W_{k}(x) \Lambda^{k}-c_{j}\right)\right]_{+}-\left[\frac{\mathcal{L}^{j}}{j !}\left(\sum_{k \geq 0} W_{k}(x) \Lambda^{k}-c_{j}\right)\right]_{-} .
\end{aligned}
$$

Then one can derive the $s_{1}$ flow equation of the EZTH as

$$
\begin{gathered}
\epsilon U_{s_{1}}=(1-\Lambda)\left(V\left(\Lambda^{-1}-1\right)^{-1} \epsilon(\log V)_{x}\right)-2(\Lambda-1) V+\frac{\epsilon}{2} U_{x}^{2}+\epsilon V_{x} \\
\epsilon V_{s_{1}}=\left(\left(\Lambda^{-1}-1\right)^{-1} \epsilon V_{x} V^{-1}+2\right)(U(x-\epsilon)-U(x)) V+\epsilon V_{x} U(x-\epsilon)+\epsilon\left(U_{x}(x-\epsilon)+U_{x}(x)\right) V
\end{gathered}
$$

where $U, V$ without bracket behind them means $U(x), V(x)$ respectively. To give a linear description of the EZTH, we introduce matrix wave functions $\psi, \bar{\psi}$ in the following part.

The matrix wave functions of the EZTH are defined by

$$
\psi=W \cdot \chi, \quad \bar{\psi}=\bar{W} \cdot \bar{\chi}
$$

where

$$
\chi(z):=z^{\frac{x}{\epsilon}} \mathbb{I}_{N}, \quad \bar{\chi}(z):=z^{-\frac{x}{\epsilon}} \mathbb{I}_{N}
$$

and the "." means the action of an operator on a function. Note that $\Lambda \cdot \chi=z \chi$ and the following asymptotic expansions can be defined

$$
\begin{array}{ll}
\psi=z^{\frac{x}{\epsilon}}\left(\mathbb{I}_{N}+\omega_{1}(x) z^{-1}+\cdots\right) \psi_{0}(z), \quad \psi_{0}:=\mathrm{e}^{\sum_{j=1}^{\infty} t_{j} \frac{z^{j}}{\epsilon j}+s_{j} \frac{z^{j}}{\epsilon j}\left(\log z-c_{j}\right)}, \quad z \rightarrow \infty, \\
\bar{\psi}=z^{-\frac{x}{\epsilon}}\left(\bar{\omega}_{0}(x)+\bar{\omega}_{1}(x) z+\cdots\right) \bar{\psi}_{0}(z), \quad \bar{\psi}_{0}:=\mathrm{e}^{\sum_{j=0}^{\infty} t_{j} \frac{z^{-j}}{\epsilon j !}+s_{j} \frac{z^{-j}}{\epsilon j !}\left(\log z-c_{j}\right)}, \quad z \rightarrow 0 .
\end{array}
$$

We can further get linear equations in the following proposition. 
Proposition 3. The matrix wave functions $\psi, \bar{\psi}$ are subject to the following Sato equations

$$
\begin{array}{rlrl}
\mathcal{L} \cdot \psi & =z \psi, & \mathcal{L} \cdot \bar{\psi}=z \bar{\psi} \\
\epsilon \partial_{t_{j}} \psi & =\left(B_{j}\right)_{+} \cdot \psi, & \epsilon \partial_{t_{j}} \bar{\psi}=\left(B_{j}\right)_{+} \cdot \bar{\psi}, \\
\epsilon \partial_{s_{j}} \psi=\left(\frac{\mathcal{L}^{j}}{\epsilon j !}\left(\log _{+} \mathcal{L}-c_{j}\right)-\left(D_{j}\right)_{-}\right) \cdot \psi, & \epsilon \partial_{s_{j}} \bar{\psi}=\left(-\frac{\mathcal{L}^{j}}{\epsilon j !}\left(\log _{-} \mathcal{L}-c_{j}\right)+\left(D_{j}\right)_{+}\right) \cdot \bar{\psi} .
\end{array}
$$

\section{Hirota bilinear equations}

From Lax equations, one can find the $s_{0}$ flow is equivalent to the spatial flow $\partial_{x}$. Basing on this fact, Hirota bilinear equations which are equivalent to the Lax equations of the EZTH can be derived in the following proposition.

Proposition 4. $W$ and $\bar{W}$ are matrix-valued wave operators of the extended $Z_{N}$-Toda hierarchy if and only the following Hirota bilinear equations hold

$$
W \Lambda^{r} W^{-1}=\bar{W} \Lambda^{-r} \bar{W}^{-1}, r \in \mathbb{N} .
$$

Proof. $\Rightarrow$ Set

$$
\alpha=\left(\alpha_{0}, \alpha_{1}, \alpha_{2}, \ldots ;\right), \beta=\left(\beta_{1}, \beta_{2}, \ldots\right),
$$

be a multi index and

$$
\partial^{\alpha}:=\partial_{t_{0}}^{\alpha_{0}} \partial_{t_{0}}^{\alpha_{1}} \partial_{t_{2}}^{\alpha_{2}} \ldots, \partial^{\beta}:=\partial_{s_{1}}^{\beta_{1}} \partial_{s_{2}}^{\beta_{2}} \ldots
$$

Suppose $\partial^{\theta}=\partial^{\alpha} \partial^{\beta}$. Firstly we shall prove the left statement leads to

$$
W(x, t, \Lambda) \Lambda^{r} W^{-1}\left(x, t^{\prime}, \Lambda\right)=\bar{W}(x, t, \Lambda) \Lambda^{-r} \bar{W}^{-1}\left(x, t^{\prime}, \Lambda\right)
$$

for all integers $r \geq 0$. Using the same as the method used in [7,9], by induction on $\alpha$, we shall prove that

$$
W(x, t, \Lambda) \Lambda^{r}\left(\partial^{\theta} W^{-1}(x, t, \Lambda)\right)=\bar{W}(x, t, \Lambda) \Lambda^{-r}\left(\partial^{\theta} \bar{W}^{-1}(x, t, \Lambda)\right) .
$$

When $\theta=0$, it is obviously true according to the definition of matrix-valued wave operators. Suppose eq.(4.5) is true in the case of $\theta \neq 0$. Note that

$$
\epsilon \partial_{p_{j}} W:= \begin{cases}{\left[\left(\partial_{t_{j}} S\right) S^{-1}+S \Lambda^{j} S^{-1}\right] W,} & p_{j}=t_{j}, \\ {\left[\left(\partial_{s_{j}} S\right) S^{-1}+S \Lambda^{j} \partial_{x} S^{-1}\right] W,} & p_{j}=s_{j},\end{cases}
$$

and

$$
\epsilon \partial_{p_{j}} \bar{W}:= \begin{cases}\left(\partial_{t_{j}} \bar{S}\right) \bar{S}^{-1} \bar{W}, & p_{j}=t_{j} \\ {\left[\left(\partial_{\bar{S}_{j}} \bar{S}\right) \bar{S}^{-1}+\bar{S} \Lambda^{-j} \partial_{x} \bar{S}^{-1}\right] \bar{W},} & p_{j}=s_{j}\end{cases}
$$

which further lead to

$$
\epsilon \partial_{p_{j}} W:= \begin{cases}\left(B_{j}\right)_{+} W, & p_{j}=t_{j} \\ {\left[-\left(D_{j}\right)_{-}+\frac{\mathcal{L}^{j}}{\epsilon j !}\left(\log _{+} \mathcal{L}-c_{j}\right)\right] W,} & p_{j}=s_{j}\end{cases}
$$

and

$$
\epsilon \partial_{p_{j}} \bar{W}:=\left\{\begin{array}{cc}
\left(B_{j}\right)_{+} \bar{W}, & p_{j}=t_{j} \\
{\left[\left(D_{j}\right)_{+}-\frac{\mathcal{L}^{j}}{\epsilon j !}\left(\log _{-} \mathcal{L}-c_{j}\right)\right] \bar{W},} & p_{j}=s_{j} \\
8 &
\end{array}\right.
$$


This further implies

$$
\left(\partial_{p_{j}} W\right) \Lambda^{r}\left(\partial^{\theta} W^{-1}\right)=\left(\partial_{p_{j}} \bar{W}\right) \Lambda^{-r}\left(\partial^{\theta} \bar{W}^{-1}\right)
$$

by considering (4.5) and furthermore we get

$$
W \Lambda^{r}\left(\partial_{p_{j}} \partial^{\theta} W^{-1}\right)=\bar{W} \Lambda^{-r}\left(\partial_{p_{j}} \partial^{\theta} \bar{W}^{-1}\right) .
$$

Thus if we increase the power of $\partial_{p_{j}}$ by 1, eq.(4.5) still holds. The induction is completed. Taylor expanding both sides of eq.(4.4) about $t=t^{\prime}, s=s^{\prime}$, one can finish the proof of eq.(4.4).

$\Leftarrow$ Vice versa, by separating the negative and the positive part of the equation, we can prove $S, \bar{S}$ are a pair of matrix-valued wave operators.

To give a description in terms of matrix-valued wave functions, the following symbolic definitions are needed.

If the series have forms

$$
\begin{gathered}
W(x, t, s, \Lambda)=\sum_{i \in \mathbb{Z}} a_{i}\left(x, t, s, \partial_{x}\right) \Lambda^{i} \text { and } \bar{W}(x, t, s, \Lambda)=\sum_{i \in \mathbb{Z}} b_{i}\left(x, t, s, \partial_{x}\right) \Lambda^{i}, \\
W^{-1}(x, t, s, \Lambda)=\sum_{i \in \mathbb{Z}} \Lambda^{i} a_{i}^{\prime}\left(x, t, s, \partial_{x}\right) \text { and } \bar{W}^{-1}(x, t, s, \Lambda)=\sum_{j \in \mathbb{Z}} \Lambda^{j} b_{j}^{\prime}\left(x, t, s, \partial_{x}\right),
\end{gathered}
$$

then we denote their corresponding left symbols $\mathcal{W}, \overline{\mathcal{W}}$ and right symbols $\mathcal{W}^{-1}, \overline{\mathcal{W}}^{-1}$ as follows

$$
\begin{aligned}
& \mathcal{W}(x, t, s, \lambda)=\sum_{i \in \mathbb{Z}} a_{i}\left(x, t, s, \partial_{x}\right) \lambda^{i}, \quad \mathcal{W}^{-1}(x, t, s, \lambda)=\sum_{i \in \mathbb{Z}} a_{i}^{\prime}\left(x, t, s, \partial_{x}\right) \lambda^{i}, \\
& \overline{\mathcal{W}}(x, t, s, \lambda)=\sum_{i \in \mathbb{Z}} b_{i}\left(x, t, s, \partial_{x}\right) \lambda^{i}, \quad \overline{\mathcal{W}}^{-1}(x, t, s, \bar{t}, \lambda)=\sum_{j \in \mathbb{Z}} b_{j}^{\prime}\left(x, t, s, \partial_{x}\right) \lambda^{j} .
\end{aligned}
$$

With above preparation, it is time to give another form of Hirota bilinear equation(see the following proposition) after defining residue as $\operatorname{Res}_{\lambda} \sum_{n \in \mathbb{Z}} \alpha_{n} \lambda^{n}=\alpha_{-1}$ using the similar proof as $[3,7,9$.

Proposition 5. Let $s_{0}=s_{0}^{\prime}, S$ and $\bar{S}$ are matrix-valued wave operators of the $Z_{N}$-Toda hierarchy if and only if for all $m \in \mathbb{Z}, r \in \mathbb{N}$, the following Hirota bilinear identity hold

$$
\begin{aligned}
& \operatorname{Res}_{\lambda}\left\{\lambda^{r+m-1} \mathcal{W}\left(x, t, s, \epsilon \partial_{x}, \lambda\right) \mathcal{W}^{-1}\left(x-m \epsilon, t^{\prime}, s^{\prime}, \epsilon \partial_{x}, \lambda\right)\right\}= \\
& \operatorname{Res}_{\lambda}\left\{\lambda^{-r+m-1} \overline{\mathcal{W}}\left(x, t, s, \epsilon \partial_{x}, \lambda\right) \overline{\mathcal{W}}^{-1}\left(x-m \epsilon, t^{\prime}, s^{\prime}, \epsilon \partial_{x}, \lambda\right)\right\}
\end{aligned}
$$

Proof. Let $m \in \mathbb{Z}, r \in \mathbb{N}$ and $s_{0}=s_{0}^{\prime}$. Put

$$
\begin{aligned}
W(x, t, s, \Lambda) & =\sum_{i \in \mathbb{Z}} a_{i}\left(x, t, s, \partial_{x}\right) \Lambda^{i} \text { and } \bar{W}(x, t, s, \Lambda)=\sum_{i \in \mathbb{Z}} b_{i}\left(x, t, s, \partial_{x}\right) \Lambda^{i}, \\
W^{-1}(x, t, s, \Lambda) & =\sum_{i \in \mathbb{Z}} \Lambda^{i} a_{i}^{\prime}\left(x, t, s, \partial_{x}\right) \text { and } \bar{W}^{-1}(x, t, \Lambda)=\sum_{j \in \mathbb{Z}} \Lambda^{j} b_{j}^{\prime}\left(x, t, s, \partial_{x}\right),
\end{aligned}
$$

and compare the coefficients in front of $\Lambda^{-m}$ in eq.(4.4):

$$
\sum_{i+j=-m-r} a_{i}\left(x, t, s, \partial_{x}\right) a_{j}^{\prime}\left(x-m \epsilon, t^{\prime}, s^{\prime}, \partial_{x}\right)=\sum_{i+j=-m+r} b_{i}\left(x, t, s, \partial_{x}\right) b_{j}^{\prime}\left(x-m \epsilon, t^{\prime}, s^{\prime}, \partial_{x}\right) .
$$

This equality can be written also as eq.(4.6). 
To give Hirota quadratic function in terms of tau functions, we need to define and prove the existence of tau function of the EZTH firstly in the next section.

\section{TAu-Functions of EZTH}

Introduce the following sequences:

$$
t-[\lambda]:=\left(t_{j}-\epsilon(j-1) ! \lambda^{j}, 0 \leq j \leq \infty\right) .
$$

A matrix-valued function $\tau \in Z_{N}$ depending only on the dynamical variables $t$ and $\epsilon$ is called the Matrix tau-function of the EZTH if it provides symbols related to matrix-valued wave operators as following,

$$
\begin{aligned}
\mathbb{S}: & =\frac{\tau\left(s_{0}+x-\frac{\epsilon}{2}, t_{j}-\frac{\epsilon(j-1) !}{\lambda^{j}}, s ; \epsilon\right)}{\tau\left(s_{0}+x-\frac{\epsilon}{2}, t, s ; \epsilon\right)}, \\
\mathbb{S}^{-1}: & =\frac{\tau\left(s_{0}+x+\frac{\epsilon}{2}, t_{j}+\frac{\epsilon(j-1) !}{\lambda^{j}}, s ; \epsilon\right)}{\tau\left(s_{0}+x+\frac{\epsilon}{2}, t, s ; \epsilon\right)}, \\
\overline{\mathbb{S}}: & =\frac{\tau\left(s_{0}+x+\frac{\epsilon}{2}, t_{j}+\epsilon(j-1) ! \lambda^{j}, s ; \epsilon\right)}{\tau\left(s_{0}+x-\frac{\epsilon}{2}, t, s ; \epsilon\right)}, \\
\overline{\mathbb{S}}^{-1}: & =\frac{\tau\left(s_{0}+x-\frac{\epsilon}{2}, t_{j}-\epsilon(j-1) ! \lambda^{j}, s ; \epsilon\right)}{\tau\left(s_{0}+x+\frac{\epsilon}{2}, t, s ; \epsilon\right)} .
\end{aligned}
$$

Here the division means the multiplication of the numerator matrix by the inverse of the denominator matrix. One can get the solution $U, V$ in terms of tau functions as

$$
U=(\log \tau)_{x x}, V=\log \frac{\tau(x+\epsilon) \tau(x-\epsilon)}{\tau^{2}(x)}
$$

When $N=2$

$$
\begin{aligned}
& {\left[\begin{array}{cc}
u_{0} & 0 \\
u_{1} & u_{0}
\end{array}\right]=\left[\begin{array}{cc}
\left(\log \tau_{0}\right)_{x x} & 0 \\
\left(\frac{\tau_{1}}{\tau_{0}}\right)_{x x} & \left(\log \tau_{0}\right)_{x x}
\end{array}\right],} \\
& e^{\left[\begin{array}{cc}
v_{0} & 0 \\
v_{1} & v_{0}
\end{array}\right]}=\left[\begin{array}{cc}
e^{v_{0}} & 0 \\
v_{1} e^{v_{0}} & e^{v_{0}}
\end{array}\right] \\
& =\left[\begin{array}{cc}
\frac{\tau_{0}(x+\epsilon) \tau_{0}(x-\epsilon)}{\tau_{0}} & 0 \\
\frac{\tau_{1}(x+\epsilon) \tau_{0}(x-\epsilon)+\tau_{0}(x+\epsilon) \tau_{1}(x-\epsilon)}{\tau_{0}}-\frac{\tau_{0}(x+\epsilon) \tau_{0}(x-\epsilon) \tau_{1}}{\tau_{0}^{2}} & \frac{\tau_{0}(x+\epsilon) \tau_{0}(x-\epsilon)}{\tau_{0}}
\end{array}\right] .
\end{aligned}
$$

This implies

$$
\begin{aligned}
& u_{0}=\left(\log \tau_{0}\right)_{x x}, \quad u_{1}=\left(\frac{\tau_{1}}{\tau_{0}}\right)_{x x}, \\
& v_{0}=\log \frac{\tau_{0}(x+\epsilon) \tau_{0}(x-\epsilon)}{\tau_{0}}, \quad v_{1}=\left(\Lambda-1+\Lambda^{-1}\right) \frac{\tau_{1}(x)}{\tau_{0}(x)} .
\end{aligned}
$$

By the Proposition 5, one can prove the following lemma 
Lemma 2. The following equations hold

$$
\begin{aligned}
& \sum_{k=1}^{N} \mathbb{S}\left(x, t, \lambda_{1}\right)_{i k} \mathbb{S}^{-1}\left(x+\epsilon, t+\left[\lambda_{2}\right], \lambda_{1}\right)_{k j}=\sum_{k=1}^{N} \overline{\mathbb{S}}\left(x, t, \lambda_{2}\right)_{i k} \overline{\mathbb{S}}^{-1}\left(x, t-\left[\lambda_{1}^{-1}\right], \lambda_{2}\right)_{k j}, \\
& \sum_{k=1}^{N} \mathbb{S}\left(x, t, \lambda_{1}\right)_{i k} \mathbb{S}^{-1}\left(x, t-\left[\lambda_{2}^{-1}\right], \lambda_{1}\right)_{k j}=\sum_{k=1}^{N} \mathbb{S}\left(x, t, \lambda_{2}\right)_{i k} \mathbb{S}^{-1}\left(x, t-\left[\lambda_{1}^{-1}\right], \lambda_{2}\right)_{k j}, \\
& \sum_{k=1}^{N} \overline{\mathbb{S}}\left(x, t, \lambda_{1}\right)_{i k} \overline{\mathbb{S}}^{-1}\left(x+\epsilon, t+\left[\lambda_{2}\right], \lambda_{1}\right)_{k j}=\sum_{k=1}^{N} \overline{\mathbb{S}}\left(x, t, \lambda_{2}\right)_{i k} \overline{\mathbb{S}}^{-1}\left(x+\epsilon, t+\left[\lambda_{1}\right], \lambda_{2}\right)_{k j} .
\end{aligned}
$$

Using Lemma 2, we can prove the following important proposition which gives the existence of matrix-valued tau functions.

Proposition 6. Given a pair of wave operators $\mathbb{S}$ and $\overline{\mathbb{S}}$ of the EZTH there exists corresponding matrix-valued invertible tau-functions $\tau \in Z_{N}$, which is unique up to the multiplication by a non-vanishing function independent of $t_{j}, j \geq 1$.

Proof. Here, we shall note that the $Z_{N}$-valued tau function $\tau(x, \mathbf{t})$ corresponding to the wave operators $\mathbb{S}$ and $\overline{\mathbb{S}}$ is in fact $\tau(x-\epsilon / 2, \mathbf{t})$.

The system is equivalent to:

$$
\begin{aligned}
& \log \mathbb{S}=\left(\exp \left(-\epsilon \sum_{j=0}^{\infty} j ! \lambda^{-(j+1)} \partial_{t_{j}}\right)-1\right) \log \tau \\
& \log \overline{\mathbb{S}}=\left(\exp \left(\epsilon \partial_{x}+\epsilon \sum_{j=0}^{\infty} j ! \lambda^{j+1} \partial_{t_{j}}\right)-1\right) \log \tau, \\
& \partial_{s_{0}} \log \tau(x, \mathbf{t})=\partial_{x} \log \tau(x, \mathbf{t}) .
\end{aligned}
$$

Then using Lemma 2 will help us to derive the existence of the tau function of this hierarchy.

After giving tau functions of the EZTH, what is the Hirota bilinear equation in form of tau function becomes a natural question which will be answered in the next section with the help of generalized Vertex operators.

\section{Generalized matrix Vertex operators and Hirota quadratic equations}

In this section we continue to discuss on the fundamental properties of the tau function of the EZTH, i.e., the Hirota quadratic equations of the EZTH. So we introduce the following vertex operators

$$
\begin{aligned}
& \Gamma^{ \pm a}:=\exp \left( \pm \frac{1}{\epsilon}\left(\sum_{j=0}^{\infty} t_{j} \frac{\lambda^{j+1}}{(j+1) !}+s_{j} \frac{\lambda^{j}}{j !}\left(\log \lambda-c_{j}\right)\right)\right) \times \exp \left(\mp \frac{\epsilon}{2} \partial_{s_{0}} \mp\left[\lambda^{-1}\right]_{\partial}\right) \\
& \Gamma^{ \pm b}:=\exp \left( \pm \frac{1}{\epsilon}\left(\sum_{j=0}^{\infty} t_{j} \frac{\lambda^{-j-1}}{(j+1) !}-s_{j} \frac{\lambda^{-j}}{j !}\left(\log \lambda-c_{j}\right)\right)\right) \times \exp \left(\mp \frac{\epsilon}{2} \partial_{s_{0}} \mp[\lambda]_{\partial}\right)
\end{aligned}
$$

where

$$
[\lambda]_{\partial}:=\epsilon \sum_{\substack{j=0 \\ 11}}^{\infty} j ! \lambda^{j+1} \partial_{t_{j}} .
$$


Because of the logarithm $\log \lambda$, the vertex operators $\Gamma^{ \pm a} \otimes \Gamma^{\mp a}$ and $\Gamma^{ \pm b} \otimes \Gamma^{\mp b}$ are multi-valued function. There are monodromy factors $M^{a}$ and $M^{b}$ respectively as following among different branches around $\lambda=\infty$

$$
\begin{aligned}
& M^{a}=\exp \left\{ \pm \frac{2 \pi i}{\epsilon} \sum_{j \geq 0} \frac{\lambda^{j}}{j !}\left(s_{j} \otimes 1-1 \otimes s_{j}\right)\right\} \\
& M^{b}=\exp \left\{ \pm \frac{2 \pi i}{\epsilon} \sum_{j \geq 0} \frac{\lambda^{-j}}{j !}\left(s_{j} \otimes 1-1 \otimes s_{j}\right)\right\} .
\end{aligned}
$$

In order to offset the complication, we need to generalize the concept of Vertex operators which leads it to be not scalar-valued any more but take values in a differential operator algebra in $Z_{N}$. So we introduce the following vertex operators

$$
\Gamma_{a}=\exp \left(-\sum_{j>0} \frac{j ! \lambda^{j+1}}{\epsilon}\left(\epsilon \partial_{x}\right) s_{j}\right) \exp \left(x \partial_{s_{0}}\right)
$$

$$
\Gamma_{b}=\exp \left(-\sum_{j>0} \frac{j ! \lambda^{-(j+1)}}{\epsilon}\left(\epsilon \partial_{x}\right) s_{j}\right) \exp \left(x \partial_{s_{0}}\right)
$$

$$
\begin{aligned}
& \Gamma_{a}^{\#}=\exp \left(x \partial_{s_{0}}\right) \exp \left(\sum_{j>0} \frac{j ! \lambda^{j+1}}{\epsilon}\left(\epsilon \partial_{x}\right) s_{j}\right) \\
& \Gamma_{b}^{\#}=\exp \left(x \partial_{s_{0}}\right) \exp \left(\sum_{j>0} \frac{j ! \lambda^{-(j+1)}}{\epsilon}\left(\epsilon \partial_{x}\right) s_{j}\right) .
\end{aligned}
$$

Then

$$
\begin{gathered}
\Gamma_{a}^{\#} \otimes \Gamma_{a}=\exp \left(x \partial_{s_{0}}\right) \exp \left(\sum_{j>0} \frac{j ! \lambda^{j+1}}{\epsilon}\left(\epsilon \partial_{x}\right)\left(s_{j}-s_{j}^{\prime}\right)\right) \exp \left(x \partial_{s_{0}^{\prime}}\right), \\
\Gamma_{b}^{\#} \otimes \Gamma_{b}=\exp \left(x \partial_{s_{0}}\right) \exp \left(\sum_{j>0} \frac{j ! \lambda^{-(j+1)}}{\epsilon}\left(\epsilon \partial_{x}\right)\left(s_{j}-s_{j}^{\prime}\right)\right) \exp \left(x \partial_{s_{0}^{\prime}}\right) .
\end{gathered}
$$

After some computation we get

$$
\begin{aligned}
& \left(\Gamma_{a}^{\#} \otimes \Gamma_{a}\right) M^{a}=\exp \left\{ \pm \frac{2 \pi i}{\epsilon} \sum_{j>0} \frac{\lambda^{j}}{j !}\left(s_{j}-s_{j}^{\prime}\right)\right\} \\
& \exp \left( \pm \frac{2 \pi i}{\epsilon}\left(\left(s_{0}+x\right)-\left(s_{0}^{\prime}+x+\sum_{j>0} \frac{\lambda^{j}}{j !}\left(s_{j}-s_{j}^{\prime}\right)\right)\right)\left(\Gamma_{a}^{\#} \otimes \Gamma_{a}\right)\right. \\
= & \exp \left( \pm \frac{2 \pi i}{\epsilon}\left(s_{0}-s_{0}^{\prime}\right)\right)\left(\Gamma_{a}^{\#} \otimes \Gamma_{a}\right),
\end{aligned}
$$




$$
\begin{aligned}
& \left(\Gamma_{b}^{\#} \otimes \Gamma_{b}\right) M^{b}=\exp \left\{ \pm \frac{2 \pi i}{\epsilon} \sum_{j>0} \frac{\lambda^{-j}}{j !}\left(s_{j}-s_{j}^{\prime}\right)\right\} \\
& \exp \left( \pm \frac{2 \pi i}{\epsilon}\left(\left(s_{0}+x\right)-\left(s_{0}^{\prime}+x+\sum_{j>0} \frac{\lambda^{-j}}{j !}\left(s_{j}-s_{j}^{\prime}\right)\right)\right)\left(\Gamma_{b}^{\#} \otimes \Gamma_{b}\right)\right. \\
= & \exp \left( \pm \frac{2 \pi i}{\epsilon}\left(s_{0}-s_{0}^{\prime}\right)\right)\left(\Gamma_{b}^{\#} \otimes \Gamma_{b}\right) .
\end{aligned}
$$

Thus when $s_{0}-s_{0}^{\prime} \in \mathbb{Z} \epsilon,\left(\Gamma_{a}^{\#} \otimes \Gamma_{a}\right)\left(\Gamma^{a} \otimes \Gamma^{-a}\right)$ and $\left(\Gamma_{b}^{\#} \otimes \Gamma_{b}\right)\left(\Gamma^{-b} \otimes \Gamma^{b}\right)$ are all single-valued near $\lambda=\infty$.

Now we should note that the above vertex operators take value in a $Z_{N}$-valued differential operator algebra $\mathbb{C}[\partial, x, t, s, \epsilon]:=\left\{f(x, t, \epsilon) \mid f(x, t, s, \epsilon)=\sum_{i \geq 0} \sum_{k \geq 0}^{N} c_{i k}(x, t, s, \epsilon) \Gamma^{k} \partial^{i}\right\}$.

Theorem 1. The invertible $Z_{N}$-valued matrix $\tau(t, s, \epsilon)$ is a tau-function of the EZTH if and only if it satisfies the following Hirota quadratic equations of the EZTH,

$$
\operatorname{Res}_{\lambda} \lambda^{r-1}\left(\Gamma_{a}^{\#} \otimes \Gamma_{a}\right)\left(\Gamma^{a} \otimes \Gamma^{-a}\right)(\tau \otimes \tau)=\operatorname{Res}_{\lambda} \lambda^{-r-1}\left(\Gamma_{b}^{\#} \otimes \Gamma_{b}\right)\left(\Gamma^{-b} \otimes \Gamma^{b}\right)(\tau \otimes \tau)
$$

computed at $s_{0}-s_{0}^{\prime}=l \epsilon$ for each $l \in \mathbb{Z}, r \in \mathbb{N}$.

Proof. We just need to prove that the HBEs are equivalent to the right side in Proposition 5 . By a straightforward computation we can get the following four identities

$$
\begin{aligned}
\Gamma_{a}^{\#} \Gamma^{a} \tau & =\tau\left(s_{0}+x-\epsilon / 2, t, s\right) \lambda^{s_{0} / \epsilon} \mathcal{W}\left(x, t, s, \epsilon \partial_{x}, \lambda\right) \lambda^{\mathbb{I}_{N} x / \epsilon}, \\
\Gamma_{a} \Gamma^{-a} \tau & =\lambda^{-\left(s_{0}+x\right) / \epsilon} \mathcal{W}^{-1}\left(x, t, s, \epsilon \partial_{x}, \lambda\right) \tau\left(x+s_{0}+\epsilon / 2, t, s\right) \\
\Gamma_{b}^{\#} \Gamma^{-b} \tau & =\tau\left(x+s_{0}-\epsilon / 2, t, s\right) \lambda^{s_{0} / \epsilon} \overline{\mathcal{W}}\left(x, t, s, \epsilon \partial_{x}, \lambda\right) \lambda^{x \mathbb{I}_{N} / \epsilon}, \\
\Gamma_{b} \Gamma^{b} \bar{\tau} & =\lambda^{-s_{0} / \epsilon} \lambda^{-x \mathbb{I}_{N} / \epsilon} \overline{\mathcal{W}}^{-1}\left(x, t, s, \epsilon \partial_{x}, \lambda\right) \tau\left(x+s_{0}+\epsilon / 2, t, s\right) .
\end{aligned}
$$

The proof of four equations eq.(6.10)-eq.(6.13) can be derived by a similar method as in [7, 9 . By substituting four equations eq.(6.10)-eq.(6.13) into the HBEs (6.9), eq.(4.6) is derived.

Doing a transformation on the eq.(6.9) by $\lambda \rightarrow \lambda^{-1}$, then the eq.(6.9) becomes

$$
\operatorname{Res}_{\lambda} \lambda^{r-1}\left(\left(\Gamma_{a}^{\#} \otimes \Gamma_{a}\right)\left(\Gamma^{a} \otimes \Gamma^{-a}-\Gamma^{-a} \otimes \Gamma^{a}\right)\right)(\tau \otimes \tau)=0
$$

computed at $s_{0}-s_{0}^{\prime}=l \epsilon$ for each $l \in \mathbb{Z}, r \in \mathbb{N}$. That means

$$
\frac{d \lambda}{\lambda}\left(\left(\Gamma_{a}^{\#} \otimes \Gamma_{a}\right)\left(\Gamma^{a} \otimes \Gamma^{-a}-\Gamma^{-a} \otimes \Gamma^{a}\right)\right)(\tau \otimes \tau)
$$

is regular in $\lambda$ computed at $s_{0}-s_{0}^{\prime}=l \epsilon$ for each $l \in \mathbb{Z}$. The eq. (6.15) in the case when $N=1$ is exactly the Hirota quadratic equation (1.2) of the extended Toda hierarchy in [7]. As we know, the Vertex operator in fact gives one special Backlund transformation of the EZTH. To give more information on the relations among different solutions of the EZTH, the Darboux transformation of the EZTH will be constructed using kernel determinant technique as [24, 25] in the next section. 


\section{Darboux transformations of the EZTH}

In this section, we will consider the Darboux transformation of the EZTH on the Lax operator

$$
\mathcal{L}=\Lambda+U+V \Lambda^{-1},
$$

i.e.

$$
\mathcal{L}^{[1]}=\Lambda+U^{[1]}+V^{[1]} \Lambda^{-1}=W \mathcal{L} W^{-1},
$$

where $W$ is the Darboux transformation operator. That means after Darboux transformation, the spectral problem about $N \times N$ matrix-valued $\phi$

$$
\mathcal{L} \phi=\Lambda \phi+U \phi+V \Lambda^{-1} \phi=\lambda \phi,
$$

will become

$$
\mathcal{L}^{[1]} \phi^{[1]}=\lambda \phi^{[1]} .
$$

To keep the Lax pair of the EZTH invariant in Proposition 2 2, i.e.

$$
\begin{aligned}
\partial_{t_{j}} \mathcal{L}^{[1]} & =\left[\left(B_{j}^{[1]}\right)_{+}, \mathcal{L}^{[1]}\right], \partial_{s_{j}} \mathcal{L}^{[1]}=\left[\left(D_{j}^{[1]}\right)_{+}, \mathcal{L}^{[1]}\right], \quad B_{j}^{[1]}:=B_{j}\left(\mathcal{L}^{[1]}\right), D_{j}^{[1]}:=D_{j}\left(\mathcal{L}^{[1]}\right), \\
\partial_{t_{j}} \log \mathcal{L}^{[1]} & =\left[\left(B_{j}^{[1]}\right)_{+}, \log \mathcal{L}^{[1]}\right], \quad\left(\log \mathcal{L}^{[1]}\right)_{s_{j}}=\left[-\left(D_{j}^{[1]}\right)_{-}, \log _{+} \mathcal{L}^{[1]}\right]+\left[\left(D_{j}^{[1]}\right)_{+}, \log _{-} \mathcal{L}^{[1]}\right],
\end{aligned}
$$

the dressing operator $W$ should satisfy the following dressing equation

$$
W_{t_{j}}=-W\left(B_{j}\right)_{+}+\left(W B_{j} W^{-1}\right)_{+} W, \quad j \geq 0 .
$$

where $W_{t_{j}}$ means the derivative of $W$ by $t_{j}$. $W$ should also satisfy the following dressing equation

$$
W_{s_{j}}=-W\left(D_{j}\right)_{+}+\left(W D_{j} W^{-1}\right)_{+} W, \quad j \geq 0 .
$$

where $W_{s_{j}}$ means the derivative of $W$ by $s_{j}$.

Now, we will give the following important theorem which will be used to generate new solutions.

Theorem 2. If $\phi$ is the first wave function of the EZTH, the Darboux transformation operator of the EZTH

$$
W(\lambda)=\left(1-\phi(\phi(x-\epsilon))^{-1} \Lambda^{-1}\right)=\phi \circ\left(1-\Lambda^{-1}\right) \circ \phi^{-1},
$$

will generater new solutions $U^{[1]}, V^{[1]}$ from seed solutions $U, V$

$$
\begin{aligned}
& U^{[1]}=U+(\Lambda-1) \phi(\phi(x-\epsilon))^{-1}, \\
& V^{[1]}=\Lambda^{-1} V \frac{\phi \Lambda^{-2} \phi}{\Lambda^{-1} \phi^{2}} .
\end{aligned}
$$

Define $\phi_{i}=\phi_{i}^{[0]}:=\left.\phi\right|_{\lambda=\lambda_{i}}$, then one can choose the specific one-fold Darboux transformation of the EZTH as following

$$
W_{1}\left(\lambda_{1}\right)=\mathbb{I}_{N}-\phi_{1}\left(\phi_{1}(x-\epsilon)\right)^{-1} \Lambda^{-1} .
$$

Meanwhile, we can also get Darboux transformation on wave function $\phi$ as following

$$
\phi^{[1]}=\left(\mathbb{I}_{N}-\phi_{1}(x)\left(\phi_{1}(x-\epsilon)\right)^{-1} \Lambda^{-1}\right) \phi .
$$


Then using iteration on Darboux transformation, the $j$-th Darboux transformation from the $(j-1)$-th solution is as

$$
\begin{aligned}
\phi^{[j]} & =\left(\mathbb{I}_{N}-\frac{\phi_{j}^{[j-1]}}{\Lambda^{-1} \phi_{j}^{[j-1]}} \Lambda^{-1}\right) \phi^{[j-1]}, \\
U^{[j]} & =U^{[j-1]}+(\Lambda-1) \frac{\phi_{j}^{[j-1]}}{\Lambda^{-1} \phi_{j}^{[j-1]}}, \\
V^{[j]} & =\left(\Lambda^{-1} V^{[j-1]}\right) \frac{\phi_{j}^{[j-1]}}{\Lambda^{-1} \phi_{j}^{[j-1]}} \frac{\Lambda^{-2} \phi_{j}^{[j-1]}}{\Lambda^{-1} \phi_{j}^{[j-1]}},
\end{aligned}
$$

where $\phi_{i}^{[j-1]}:=\left.\phi^{[j-1]}\right|_{\lambda=\lambda_{i}}$, are wave functions corresponding to different spectrals with the $(j-1)$-th solutions $U^{[j-1]}, V^{[j-1]}$. It can be checked that $\phi_{i}^{[j-1]}=0, i=1,2, \ldots, j-1$.

After iteration on Darboux transformations, we can generalize the Darboux transformation to $n$-fold case which is contained in the following theorem.

Theorem 3. The $n$-fold Darboux transformation of EZTH equation is as following

$$
W_{n}=\mathbb{I}_{N}+t_{1}^{[n]} \Lambda^{-1}+t_{2}^{[n]} \Lambda^{-2}+\cdots+t_{n}^{[n]} \Lambda^{-n}
$$

where

$$
\left.W_{n} \cdot \phi_{i}\right|_{i \leq n}=0
$$

The Darboux transformation leads to new solutions from seed solutions

$$
\begin{aligned}
U^{[n]} & =U+(\Lambda-1) t_{1}^{[n]} \\
V^{[n]} & =t_{n}^{[n]}(x)\left(\Lambda^{-n} V\right) t_{n}^{[n]-1}(x-\epsilon) .
\end{aligned}
$$

where

$$
\begin{aligned}
& \left(W_{n}\right)_{i j}=\frac{1}{\Delta_{n}}
\end{aligned}
$$

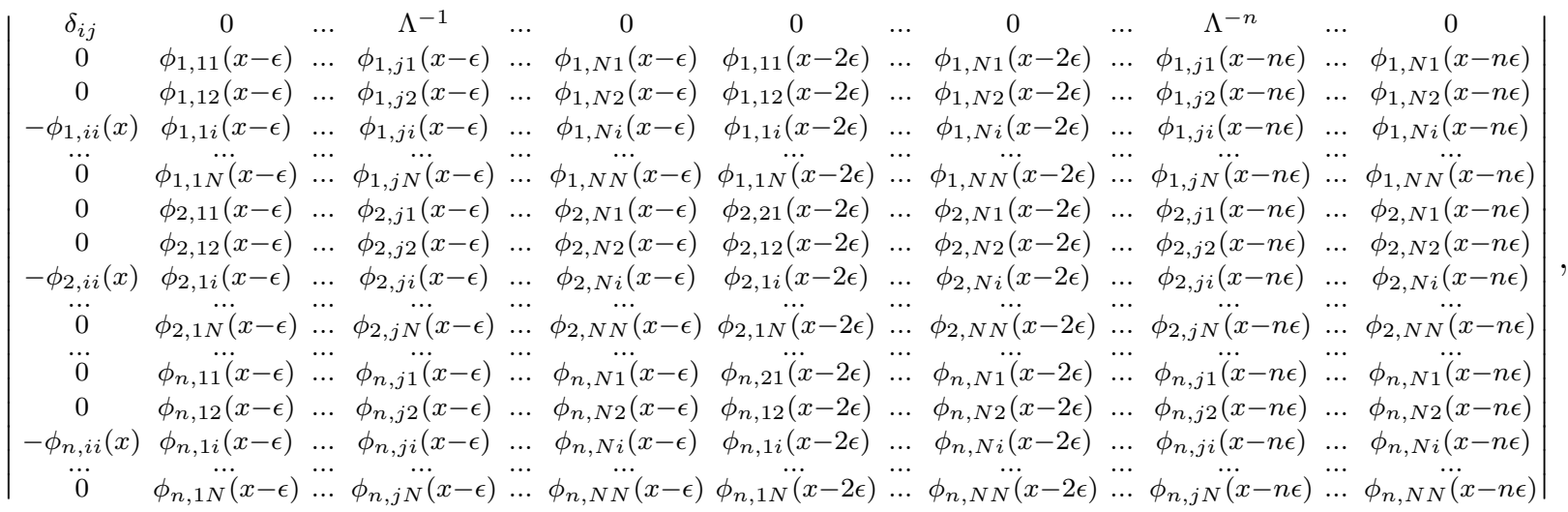

$$
\begin{aligned}
& \Delta_{n}=\left|\begin{array}{cccc}
\phi_{1}(x-\epsilon) & \phi_{1}(x-2 \epsilon) & \ldots & \phi_{1}(x-n \epsilon) \\
\phi_{2}(x-\epsilon) & \phi_{2}(x-2 \epsilon) & \ldots & \phi_{2}(x-n \epsilon) \\
\phi_{n}(\dddot{x}-\epsilon) & \phi_{n}(\dddot{x}-2 \epsilon) & \ldots & \phi_{n}(\dddot{x}-n \epsilon)
\end{array}\right| .
\end{aligned}
$$


It can be easily checked that $W_{n} \phi_{i}=0, i=1,2, \ldots, n$.

Taking seed solution $U=(0)_{N \times N}, V=\mathbb{I}_{N}$, then using Theorem [3, one can get the $n$-th new solution of the EZTH as

$$
\begin{aligned}
U^{[n]} & =\left(1-\Lambda^{-1}\right) \partial_{t_{0}} \log \bar{W}_{r}\left(\phi_{1}, \phi_{2}, \ldots \phi_{n}\right), \\
V^{[n]} & =e^{\left(1-\Lambda^{-1}\right)\left(1-\Lambda^{-1}\right) \log \bar{W}_{r}\left(\phi_{1}, \phi_{2}, \ldots \phi_{n}\right)},
\end{aligned}
$$

where $\bar{W}_{r}\left(\phi_{1}, \phi_{2}, \ldots \phi_{n}\right)$ is the "Hankel" in terms of $\Gamma$

$$
\bar{W}_{r}\left(\phi_{1}, \phi_{2}, \ldots \phi_{n}\right)=\operatorname{det}\left(\Lambda^{-j+1} \phi_{n+1-i}\right)_{1 \leq i, j \leq n} .
$$

"Hankel" in terms of $\Gamma$ means in the process of calculation, we treat every element $\phi_{n+1-i}$ not directly as a matrix form but as a scalar polynomial of element $\Gamma$. After getting the values of $U^{[n]}, V^{[n]}$ in terms of $\Gamma$, we then rewrite it in matrix form.

7.1. Soliton solutions. After the above preparation over the first Darboux transformation, in this section, we will use the first Darboux transformation of the EZTH to generate new solutions from trivial seed solutions. In particular, some matrix-valued soliton solutions will be shown using the first Darboux transformation.

For $N=2$, one can take seed solution $U=\left[\begin{array}{ll}0 & 0 \\ 0 & 0\end{array}\right], V=\left[\begin{array}{ll}1 & 0 \\ 0 & 1\end{array}\right]$, then the initial wave function $\phi_{i}$ satisfies

$$
\begin{gathered}
\Lambda \phi+\left[\begin{array}{ll}
1 & 0 \\
0 & 1
\end{array}\right] \Lambda^{-1} \phi=\left[\begin{array}{cc}
\lambda_{1} & 0 \\
\lambda_{2} & \lambda_{1}
\end{array}\right] \phi, 1 \leq i \leq n . \\
\phi=\exp \left(\frac{x}{\epsilon} \log \left[\begin{array}{cc}
z_{1} & 0 \\
z_{2} & z_{1}
\end{array}\right]\right), \quad z_{1} \neq 0,
\end{gathered}
$$

with $z_{1}+z_{1}^{-1}=\lambda_{1}, z_{2}+z_{1}^{-1}-\frac{z_{2}}{z_{1}^{2}}=\lambda_{2}$.

$$
S=E+\omega_{1} \Lambda^{-1}+v \frac{x}{\epsilon} \Lambda^{-1}-\frac{x}{\omega_{1}} \epsilon \Lambda^{-2}+\ldots, \quad \omega_{1}=\text { cons } .
$$

Under this initial equation, the operator $A_{1}$ in above Lemma 1 is in form of

$$
\begin{aligned}
A_{1}=\left(\Lambda+\left[\begin{array}{ll}
1 & 0 \\
0 & 1
\end{array}\right] \Lambda^{-1}\right) \epsilon \partial-\left(\Lambda-\left[\begin{array}{ll}
1 & 0 \\
0 & 1
\end{array}\right] \Lambda^{-1}\right) . \\
\frac{\partial \phi}{\partial s_{1}}=\left(\left[\left(\Lambda+\left[\begin{array}{ll}
1 & 0 \\
0 & 1
\end{array}\right] \Lambda^{-1}\right) \epsilon \partial-\left(\Lambda-\left[\begin{array}{ll}
1 & 0 \\
0 & 1
\end{array}\right] \Lambda^{-1}\right)\right] \phi,\right.
\end{aligned}
$$

Then solution $\phi$ in terms of $x, s_{1}$ can be chosen in the form

$$
\phi=\exp \left(\frac{x+\lambda s_{1}}{\epsilon} \log Z+\frac{s_{1}}{\epsilon}\left(-Z+Z^{-1}\right)\right), Z=\left[\begin{array}{cc}
z_{1} & 0 \\
z_{2} & z_{1}
\end{array}\right], \quad \lambda=\left[\begin{array}{cc}
\lambda_{1} & 0 \\
\lambda_{2} & \lambda_{1}
\end{array}\right]
$$

where $Z+\left[\begin{array}{cc}1 & 0 \\ 0 & 1\end{array}\right] Z^{-1}=\left[\begin{array}{cc}z_{1} & 0 \\ z_{2} & z_{1}\end{array}\right]+\left[\begin{array}{cc}1 & 0 \\ 0 & 1\end{array}\right]\left[\begin{array}{cc}z_{1}^{-1} & 0 \\ -\frac{z_{2}}{z_{1}^{2}} & z_{1}^{-1}\end{array}\right]=\lambda=\left[\begin{array}{cc}\lambda_{1} & 0 \\ \lambda_{2} & \lambda_{1}\end{array}\right]$. 


$$
\begin{aligned}
U^{[1]} & =(\Lambda-1) \frac{\cosh \left(\frac{x+\lambda s_{1}}{\epsilon} \log Z+\frac{s_{1}}{\epsilon}\left(-Z+Z^{-1}\right)\right)}{\cosh \left(\frac{x+\lambda s_{1}}{\epsilon} \log Z+\frac{s_{1}}{\epsilon}\left(-Z+Z^{-1}\right)-\log Z\right)}, \\
V^{[1]} & =\left(1-\Lambda^{-1}\right)^{2} \log \left[2 \cosh \left(\frac{x+\lambda s_{1}}{\epsilon} \log Z+\frac{s_{1}}{\epsilon}\left(-Z+Z^{-1}\right)\right)\right] .
\end{aligned}
$$

Using $\log Z=\left[\begin{array}{cc}\log z_{1} & 0 \\ \frac{z_{2}}{z_{1}} & \log z_{1}\end{array}\right], \cosh \left(\left[\begin{array}{ll}a & 0 \\ b & a\end{array}\right]\right)=\left[\begin{array}{cc}\cosh a & 0 \\ b \cosh a & \cosh a\end{array}\right]$, one can derive the specific elements in new solutions $U^{[1]}, V^{[1]}$ as

$$
\begin{aligned}
u_{0}^{[1]}= & (\Lambda-1) \frac{\cosh \left(\frac{x}{\epsilon} \log z_{1}+\frac{s_{1}}{\epsilon}\left(\lambda_{1}-z_{1}+z_{1}^{-1}\right)\right)}{\cosh \left(\frac{x-\epsilon}{\epsilon} \log z_{1}+\frac{s_{1}}{\epsilon}\left(\lambda_{1}-z_{1}+z_{1}^{-1}\right)\right)} \\
u_{1}^{[1]}= & \left(\frac{x}{\epsilon} \frac{z_{2}}{z_{1}}+\frac{s_{1}}{\epsilon}\left(\lambda_{2}-z_{2}-\frac{z_{2}}{z_{1}^{2}}\right)\right) \frac{\cosh \left(\frac{x}{\epsilon} \log z_{1}+\frac{s_{1}}{\epsilon}\left(\lambda_{1}-z_{1}+z_{1}^{-1}\right)\right)}{\cosh \left(\frac{x-\epsilon}{\epsilon} \log z_{1}+\frac{s_{1}}{\epsilon}\left(\lambda_{1}-z_{1}+z_{1}^{-1}\right)\right)} \\
& -\left(\frac{x-\epsilon}{\epsilon} \frac{z_{2}}{z_{1}}+\frac{s_{1}}{\epsilon}\left(\lambda_{2}-z_{2}-\frac{z_{2}}{z_{1}^{2}}\right)\right) \frac{\cosh \left(\frac{x}{\log } \log z_{1}+\frac{s_{1}}{\epsilon}\left(\lambda_{1}-z_{1}+z_{1}^{-1}\right)\right)}{\cosh ^{2}\left(\frac{x-\epsilon}{\epsilon} \log z_{1}+\frac{s_{1}}{\epsilon}\left(\lambda_{1}-z_{1}+z_{1}^{-1}\right)\right)} \\
v_{0}^{[1]}= & \left(1-\Lambda^{-1}\right)^{2} \log \left[2 \cosh \left(\frac{x}{\epsilon} \log z_{1}+\frac{s_{1}}{\epsilon}\left(\lambda_{1}-z_{1}+z_{1}^{-1}\right)\right)\right] \\
v_{1}^{[1]}= & \left(1-\Lambda^{-1}\right)^{2} \log \left(\frac{x}{\epsilon} \frac{z_{2}}{z_{1}}+\frac{s_{1}}{\epsilon}\left(\lambda_{2}-z_{2}-\frac{z_{2}}{z_{1}^{2}}\right)\right)
\end{aligned}
$$

Taking $z_{2}=\lambda_{2}=u_{1}^{[1]}=v_{1}^{[1]}=0$, the above soliton solutions will be reduced to soliton solutions of the scalar-valued extended Toda chain [26].

\section{Bi-Hamiltonian STRUCTURE AND TAU SYMmetry}

To describe the integrability of the EZTH, we will construct the Bi-Hamiltonian structure and tau symmetry of the EZTH in this section as [23]. For a matrix $A=\left(a_{i j}\right)=\sum_{i=0}^{N-1} a_{i} \Gamma^{i}$, the vector field $\partial_{A}$ over EZTH is defined by

$$
\partial_{A}=\sum_{i=0}^{N-1} \sum_{k \geq 0} a_{i}^{(k)}\left(\frac{\partial}{\partial u_{i}^{(k)}}+\frac{\partial}{\partial v_{i}^{(k)}}\right) .
$$

For a function $\bar{f}=\int f d x$, we have

$$
\partial_{A} \bar{f}=\int \sum_{i=0}^{N-1} \sum_{k \geq 0} a_{i}^{(k)}\left(\frac{\partial f}{\partial u_{i}^{(k)}}+\frac{\partial f}{\partial v_{i}^{(k)}}\right) d x=\int \operatorname{Tr}_{N} \sum_{k \geq 0} A^{(k)}\left(\frac{\delta f}{\delta u^{(k)}}+\frac{\delta f}{\delta v^{(k)}}\right) d x,
$$

where

$$
\left(\frac{\delta}{\delta u}\right)_{i j}=\frac{\delta}{\delta u_{j i}},\left(\frac{\delta}{\delta v}\right)_{i j}=\frac{\delta}{\delta v_{j i}},
$$


and

$$
\operatorname{Tr}_{N} A=\text { the trace of }\left[\begin{array}{cccc}
\frac{1}{N} & \frac{1}{N-1} & \cdot & 1 \\
0 & \frac{1}{N} & \cdot & \frac{1}{2} \\
\vdots & \vdots & \vdots & \vdots \\
0 & 0 & \cdot & \frac{1}{N}
\end{array}\right] A .
$$

In this section, we will consider the EZTH on Lax operator

$$
\mathcal{L}=\Lambda+u+e^{v} \Lambda^{-1}, \quad u, v \in Z_{N} .
$$

Then we can define the hamiltonian bracket as

$$
\{\bar{f}, \bar{g}\}=\int \operatorname{Tr}_{N} \sum_{w, w^{\prime}} \frac{\delta f}{\delta w}\left\{w, w^{\prime}\right\} \frac{\delta g}{\delta w^{\prime}} d x, \quad w, w^{\prime}=u_{i} \text { or } v_{j}, \quad 0 \leq i, j \leq N-1 .
$$

For $u(x)=\sum_{i=0}^{N-1} u_{i}(x) \Gamma^{i}, \quad v(x)=\sum_{i=0}^{N-1} v_{i}(x) \Gamma^{i}$, the bi-Hamiltonian structure for the EZTH can be given by the following two compatible Poisson brackets which is a generalization in matrix form of the extended Toda hierarchy in [6]

$$
\begin{aligned}
& \left\{v_{i}(x), v_{j}(y)\right\}_{1}=\left\{u_{i}(x), u_{j}(y)\right\}_{1}=0, \\
& \left\{u_{i}(x), v_{j}(y)\right\}_{1}=\frac{1}{N \epsilon} \delta_{i 0} \delta_{j 0}[\Lambda-1] \delta(x-y), \\
& \left\{u_{i}(x), u_{j}(y)\right\}_{2}=\frac{1}{N \epsilon}\left[\Lambda e^{v(x)}-e^{v(x)} \Lambda^{-1}\right]_{i+j} \delta(x-y), \\
& \left\{u_{i}(x), v_{j}(y)\right\}_{2}=\frac{1}{N \epsilon} u_{i+j}(x)[\Lambda-1] \delta(x-y), \\
& \left\{v_{i}(x), v_{j}(y)\right\}_{2}=\frac{1}{\epsilon} \delta_{i 0} \delta_{0 j}\left[\Lambda-\Lambda^{-1}\right] \delta(x-y) .
\end{aligned}
$$

For any difference operator $A=\sum_{k} A_{k} \Lambda^{k}$, define residue $\operatorname{Res} A=A_{0}$. In the following theorem, we will prove the above Poisson structure can be as the the Hamiltonian structure of the EZTH.

Theorem 4. The flows of the EZTH are Hamiltonian systems of the form

$$
\frac{\partial u_{i}}{\partial t_{k, j}}=\left\{u_{i}, H_{k, j}\right\}_{1}, \frac{\partial v_{i}}{\partial t_{k, j}}=\left\{v_{i}, H_{k, j}\right\}_{1}, \quad k=0,1 ; j \geq 0,
$$

with $t_{0, j}=t_{j}, t_{1, j}=s_{j}$. They satisfy the following bi-Hamiltonian recursion relation

$$
\left\{\cdot, H_{1, n-1}\right\}_{2}=n\left\{\cdot, H_{1, n}\right\}_{1}+2\left\{\cdot, H_{0, n-1}\right\}_{1}, \quad\left\{\cdot, H_{0, n-1}\right\}_{2}=(n+1)\left\{\cdot, H_{0, n}\right\}_{1} .
$$

Here the Hamiltonians have the form

$$
H_{k, j}=\int h_{k, j}\left(u, v ; u_{x}, v_{x} ; \ldots ; \epsilon\right) d x, \quad k=0,1 ; j \geq 0
$$

with

$$
h_{0, j}=\frac{1}{(j+1) !} \operatorname{Tr}_{N} \operatorname{Res} \mathcal{L}^{j+1}, h_{1, j}=\frac{2}{j !} \operatorname{Tr}_{N} \operatorname{Res}\left[\mathcal{L}^{j}\left(\log \mathcal{L}-c_{j}\right)\right]
$$


Proof. For $\beta=0$, i.e. the original Toda hierarchy, the proof is same as the proof in [6].

Here we will prove that the flows $\frac{\partial}{\partial t_{1, n}}$ are also Hamiltonian systems with respect to the first Poisson bracket. In [6], the following identity has been proved

(8.12) $\operatorname{Tr}_{N} \operatorname{Res}\left[\mathcal{L}^{n} d\left(S \epsilon \partial_{x} S^{-1}\right)\right] \sim \operatorname{Tr}_{N} \operatorname{Res} \mathcal{L}^{n-1} d \mathcal{L}$,

which show the validity of the following equivalence relation:

(8.13) $\operatorname{Tr}_{N} \operatorname{Res}\left(\mathcal{L}^{n} d \log _{+} \mathcal{L}\right) \sim \operatorname{Tr}_{N} \operatorname{Res}\left(\mathcal{L}^{n-1} d \mathcal{L}\right)$.

Here the equivalent relation $\sim$ is up to a $x$-derivative of another 1 -form.

In a similar way as eq.(8.12), we obtain the following equivalence relation

$$
\operatorname{Tr}_{N} \operatorname{Res}\left[\mathcal{L}^{n} d\left(\bar{S} \epsilon \partial_{x} \bar{S}^{-1}\right)\right] \sim-\operatorname{Tr}_{\mathrm{N}} \operatorname{Res} \mathcal{L}^{\mathrm{n}-1} \mathrm{~d} \mathcal{L}
$$

i.e.

(8.15) $\operatorname{Tr}_{N} \operatorname{Res}\left(\mathcal{L}^{n} d \log _{-} \mathcal{L}\right) \sim \operatorname{Tr}_{\mathrm{N}} \operatorname{Res}\left(\mathcal{L}^{\mathrm{n}-1} \mathrm{~d} \mathcal{L}\right)$.

Combining (8.13) with (8.15) together can lead to

$$
\operatorname{Tr}_{N} \operatorname{Res}\left(\mathcal{L}^{n} d \log \mathcal{L}\right) \sim \operatorname{Tr}_{N} \operatorname{Res}\left(\mathcal{L}^{\mathrm{n}-1} \mathrm{~d} \mathcal{L}\right)
$$

Suppose

$$
A_{\alpha, n}=\sum_{k} a_{\alpha, n+1 ; k} \Lambda^{k}
$$

Then from

$$
\frac{\partial \mathcal{L}}{\partial t_{k, n}}=\left[\left(B_{k, n}\right)_{+}, \mathcal{L}\right]=\left[-\left(B_{k, n}\right)_{-}, \mathcal{L}\right], \quad B_{0, n}=B_{n}, B_{1, n}=D_{n}
$$

we can derive equation

$$
\begin{aligned}
& \epsilon \frac{\partial u}{\partial t_{\beta, n}}=a_{\beta, n ; 1}(x+\epsilon)-a_{\beta, n ; 1}(x) \in Z_{N}, \beta=0,1, \\
& \epsilon \frac{\partial v}{\partial t_{\beta, n}}=a_{\beta, n ; 0}(x-\epsilon) e^{v(x)}-a_{\beta, n ; 0}(x) e^{v(x+\epsilon)} \in Z_{N} .
\end{aligned}
$$

The equivalence relation (8.13) now readily follows from the above two equations. By using (8.13) we obtain

$$
\begin{aligned}
& d \tilde{h}_{n}=\frac{2}{n !} d \operatorname{Tr}_{N} \operatorname{Res}\left[\mathcal{L}^{n}\left(\log _{+} \mathcal{L}-c_{n}\right)\right] \\
& \sim \frac{2}{(n-1) !} \operatorname{Tr}_{N} \operatorname{Res}\left[\mathcal{L}^{n-1}\left(\log _{+} \mathcal{L}-c_{n}\right) d \mathcal{L}\right]+\frac{2}{n !} \operatorname{Tr}_{N} \operatorname{Res}\left[\mathcal{L}^{n-1} d \mathcal{L}\right] \\
& =\frac{2}{(n-1) !} \operatorname{Tr}_{N} \operatorname{Res}\left[\mathcal{L}^{n-1}\left(\log _{+} \mathcal{L}-c_{n-1}\right) d \mathcal{L}\right] \\
& =\operatorname{Tr}_{N}\left[a_{1, n ; 0}(x) d u+a_{1, n ; 1}(x-\epsilon) e^{v(x)} d v\right] .
\end{aligned}
$$

It yields the following identities

$$
\frac{\delta H_{1, n}}{\delta u}=a_{1, n ; 0}(x), \quad \frac{\delta H_{1, n}}{\delta v}=a_{1, n ; 1}(x-\epsilon) e^{v(x)}
$$

This agree with Lax equation 
(8.24) $\frac{\partial u_{i}}{\partial t_{1, n}}=\left\{u_{i}, H_{1, n}\right\}_{1}=\frac{1}{\epsilon}\left[e^{\epsilon \partial_{x}}-1\right] \frac{\delta H_{1, n}}{\delta v_{i}}=\frac{1}{\epsilon}\left(a_{1, n ; 1}(x+\epsilon)-a_{1, n ; 1}(x)\right)_{i}$,

$$
\frac{\partial v_{i}}{\partial t_{1, n}}=\left\{v_{i}, H_{1, n}\right\}_{1}=\frac{1}{\epsilon}\left[1-e^{\epsilon \partial_{x}}\right] \frac{\delta H_{1, n}}{\delta u_{i}}=\frac{1}{\epsilon}\left[a_{1, n ; 0}(x-\epsilon) e^{v(x)}-a_{1, n ; 0}(x) e^{v(x+\epsilon)}\right]_{i} .
$$

From the above identities we see that the flows $\frac{\partial}{\partial t_{1, n}}$ are Hamiltonian systems of the form (8.9). For the case of $\beta=1$ the recursion relation follows from the following trivial identities

$$
\begin{aligned}
& n \frac{2}{n !} \mathcal{L}^{n}\left(\log _{ \pm} \mathcal{L}-c_{n}\right)=\mathcal{L} \frac{2}{(n-1) !} \mathcal{L}^{n-1}\left(\log _{ \pm} \mathcal{L}-c_{n-1}\right)-2 \frac{1}{n !} \mathcal{L}^{n} \\
& =\frac{2}{(n-1) !} \mathcal{L}^{n-1}\left(\log _{ \pm} \mathcal{L}-c_{n-1}\right) \mathcal{L}-2 \frac{1}{n !} \mathcal{L}^{n}
\end{aligned}
$$

Then we get, for $\beta=1$,

$$
\begin{aligned}
& n a_{1, n+1 ; 1}(x)=a_{1, n ; 0}(x+\epsilon)+u a_{1, n ; 1}(x)+e^{v} a_{1, n ; 2}(x-\epsilon)-2 a_{0, n+1 ; 1}(x) \\
& =a_{1, n ; 0}(x)+u(x+\epsilon) a_{1, n ; 1}(x)+e^{v(x+2 \epsilon)} a_{1, n ; 2}(x)-2 a_{0, n+1 ; 1}(x) .
\end{aligned}
$$

This further leads to

$$
\begin{aligned}
& \left\{u_{i}, H_{1, n-1}\right\}_{2}=\left\{\left[\Lambda e^{v(x)}-e^{v(x)} \Lambda^{-1}\right] a_{1, n ; 0}(x)+u(x)[\Lambda-1] a_{1, n ; 1}(x-\epsilon) e^{v(x)}\right\}_{i} \\
& =n\left[a_{1, n+1 ; 1}(x) e^{v(x+\epsilon)}-a_{1, n+1 ; 1}(x-\epsilon) e^{v(x)}\right]_{i}+2\left[a_{0, n+1 ; 0}(x) e^{v(x+\epsilon)}-a_{0, n+1 ; 0}(x-\epsilon) e^{v(x)}\right]_{i} .
\end{aligned}
$$

This is exactly the recursion relation on flows for $u$. The similar recursion flow on $v$ can be similarly derived. Theorem is proved till now.

Similarly as [6], the tau symmetry of the EZTH can be proved in the following theorem.

Theorem 5. The EZTH has the following tau-symmetry property:

$$
\frac{\partial h_{\alpha, m}}{\partial t_{\beta, n}}=\frac{\partial h_{\beta, n}}{\partial t_{\alpha, m}}, \quad \alpha, \beta=0,1, m, n \geq 0 .
$$

Proof. Let us prove the theorem for the case when $\alpha=1, \beta=0$, other cases are proved in a similar way

$$
\begin{aligned}
& \frac{\partial h_{1, m}}{\partial t_{0, n}}=\frac{2}{m !(n+1) !} \operatorname{Tr}_{N} \operatorname{Res}\left[-\left(\mathcal{L}^{n+1}\right)_{-}, \mathcal{L}^{m}\left(\log _{+} \mathcal{L}-c_{m}\right)\right] \\
& =\frac{2}{m !(n+1) !} \operatorname{Tr}_{N} \operatorname{Res}\left[\left(\mathcal{L}^{m}\left(\log _{+} \mathcal{L}-c_{m}\right)\right)_{+},\left(\mathcal{L}^{n+1}\right)_{-}\right] \\
& =\frac{2}{m !(n+1) !} \operatorname{Tr}_{N} \operatorname{Res}\left[\left(\mathcal{L}^{m}\left(\log _{+} \mathcal{L}-c_{m}\right)\right)_{+}, \mathcal{L}^{n+1}\right]=\frac{\partial h_{0, n}}{\partial t_{1, m}}
\end{aligned}
$$

The theorem is proved.

This property justifies the following alternative definition of another kind of tau function for the EZTH. 
Definition 3. Another tau function in $Z_{N}$ of the EZTH can be defined by the following expressions in terms of the densities of the Hamiltonians:

$$
h_{\beta, n}=\epsilon(\Lambda-1) \frac{\partial \log \bar{\tau}}{\partial t_{\beta, n}}, \quad \beta=0,1 ; n \geq 0,
$$

with $t_{0, j}=t_{j}, t_{1, j}=s_{j}$.

With above two different definitions tau functions of this hierarchy, some mysterious connections between these two kinds of tau functions become an open question. One is from the Sato theory and another is from the hamiltonian tau symmetry.

\section{Conclusions and Discussions}

In this paper, we constructed a new hierarchy called EZTH and extended Sato theory to this hierarchy including Sato equations, matrix wave operators, Hirota quadratic equations, the existence of the tau function. Similarly as extended Toda hierarchy and extended bigraded Toda hierarchy in Gromov-Witten theory of $\mathbb{C} P^{1}$ and orbiford respectively, this hierarchy deserves further studying and exploring because of its potential applications in topological quantum fields and Gromov-Witten theory. Basing on above two different definitions tau functions of this hierarchy, to derive the mysterious deep connections between these two kinds of tau functions with one defined from Sato theory and another from hamiltonian tau symmetry become an interesting question. This is not easy and will be included in our future work.

Acknowledgements: Chuanzhong Li is supported by the National Natural Science Foundation of China under Grant No. 11201251, Zhejiang Provincial Natural Science Foundation of China under Grant No. LY12A01007, the Natural Science Foundation of Ningbo under Grant No. 2013A610105, 2014A610029. Jingsong He is supported by the National Natural Science Foundation of China under Grant No. 11271210, K.C.Wong Magna Fund in Ningbo University. We would like to thank Todor E Milanov and Dafeng Zuo for their valuable discussion.

\section{REFERENCES}

[1] M. Toda, Vibration of a chain with nonlinear interaction. J. Phys. Soc. Jpn. 22(1967), 431-436.

[2] M. Toda, Nonlinear waves and solitons(Kluwer Academic Publishers, Dordrecht, Holland, 1989).

[3] K. Ueno, K. Takasaki, Toda lattice hierarchy, In "Group representations and systems of differential equations" (Tokyo, 1982), 1-95, Adv. Stud. Pure Math., 4, North-Holland, Amsterdam, 1984.

[4] E. Witten, Two-dimensional gravity and intersection theory on moduli space, Surveys in differential geometry, 1(1991), 243-310.

[5] B. A. Dubrovin, Geometry of 2D topological field theories, in Integrable systems and quantum groups (Montecatini Terme, 1993), 120-348, Lecture Notes in Math., 1620, Springer, Berlin, 1996.

[6] G. Carlet, B. Dubrovin, Y. Zhang, The Extended Toda Hierarchy, Moscow Mathematical Journal 4 (2004), 313-332.

[7] T. Milanov, Hirota quadratic equations for the extended Toda hierarchy, Duke Math. J. 138 (2007), 161-178.

[8] G. Carlet, The extended bigraded Toda hierarchy, J. Phys. A, 39 (2006), 9411-9435.

[9] C. Z. Li, J. S. He, K. Wu, Y. Cheng, Tau function and Hirota bilinear equations for the extended bigraded Toda Hierarchy, J. Math. Phys.51(2010),043514.

[10] S. Aoyama, Y. Kodama, Topological Landau-Ginzburg theory with a rational potential and the dispersionless KP hierarchy, Commun. Math. Phy. 182(1996),185-219.

[11] T. Milanov, H. H. Tseng, The spaces of Laurent polynomials, $\mathbb{P}^{1}$-orbifolds, and integrable hierarchies, Journal für die reine und angewandte Mathematik 622 (2008), 189-235.

[12] C. Z. Li, Solutions of bigraded Toda hierarchy, Journal of Physics A 44(2011), 255201. 
[13] C. Z. Li, J. S. He, Dispersionless bigraded Toda hierarchy and its additional symmetry, Reviews in Mathematical Physics, 24(2012), 1230003.

[14] C. Z. Li, J. S. He, Y. C. Su, Block type symmetry of bigraded Toda hierarchy, J. Math. Phys. 53(2012), 013517.

[15] V. G. Kac and J. W. van de Leur, The n-component KP hierarchy and representation theory, J. Math. Phys. 44 (2003), 3245.

[16] M. Adler, P. van Moerbeke and P. Vanhaecke, Moment matrices and multi-component KP, with applications to random matrix theory, Commun. Math. Phys. 286(2009), 1.

[17] M. Mañas, L. Martínez Alonso, The multicomponent 2D Toda hierarchy: dispersionless limit, Inverse Problems, 25(2009), 11.

[18] M. Mañas, L. Martínez Alonso, and C. Álvarez Fernández, The multicomponent 2D Toda hierarchy: discrete flows and string equations, Inverse Problems, 25(2009), 065007.

[19] C. Álvarez Fernández, U. Fidalgo Prieto, M. Mañas, The multicomponent 2D Toda hierarchy: generalized matrix orthogonal polynomials, multiple orthogonal polynomials and Riemann-Hilbert problems, Inverse Problems, 26(2010), 055009.

[20] C. Álvarez Fernández, U. Fidalgo Prieto, and M. Mañas 2010 Multiple orthogonal polynomials of mixed type: Gauss-Borel factorization and the multi-component 2D Toda hierarchy, Advances in Mathematics, $227(2011), 1451-1525$.

[21] G. Carlet, J. van de Leur, Hirota equations for the extended bigraded Toda hierarchy and the total descendent potential of $\mathbb{P}^{1}$ orbifolds, J. Phys. A: Math. Theor. 46(2013), 405205.

[22] C. Z. Li, J. S. He, On the extended multi-component Toda hierarchy, Math. Phys. Analyis and Geometry 17(2014), 377-407.

[23] D. F. Zuo, Local matrix generalizations of $W$-algebras, arXiv:1401.2216

[24] J. S. He, L. Zhang, Y. Cheng and Y. S. Li, Determinant representation of Darboux transformation for the AKNS system, Sci. China A, 12(2006), 1867-78.

[25] C. Z. Li, J. S. He, and K. Porsezian, Rogue waves of the Hirota and the Maxwell-Bloch equation, Physical Review E 87(2013), 012913.

[26] G. Carlet, Extended Toda hierarchy and its Hamiltonian structure, SISSA (2013), thesis. 